\title{
Velocity and Velocity-Difference Distributions in Burgers Turbulence
}

\author{
Stanislav Boldyrev, ${ }^{1}$ Timur Linde, ${ }^{1}$ and Alexandre Polyakov ${ }^{2}$ \\ ${ }^{1}$ Department of Astronomy and Astrophysics, University of Chicago, Chicago, IL 60637 \\ ${ }^{2}$ Joseph Henry Laboratories, Princeton University, Princeton, NJ 08544
}

(Dated: November 12, 2018)

\begin{abstract}
We consider the one-dimensional Burgers equation randomly stirred at large scales by a Gaussian short-time correlated force. Using the method of dissipative anomalies, we obtain velocity and velocity-difference probability density functions, and confirm the results with high-resolution numerical simulations.
\end{abstract}

PACS numbers: 47.27.-i, 47.40.-x, 52.35.Tc

1. Introduction. The one-dimensional Burgers equation with a random external force,

$$
u_{t}+u u_{x}=\nu u_{x x}+f(x, t)
$$

is a simple model of turbulence, where $u(x, t)$ is a onedimensional velocity field, and $\nu$ is small viscosity. The external force is assumed to be Gaussian, with zero mean and white-in-time covariance,

$$
\left\langle f(x, t) f\left(x^{\prime}, t^{\prime}\right)\right\rangle=\kappa\left(x-x^{\prime}\right) \delta\left(t-t^{\prime}\right),
$$

where $\kappa(y)$ should be specified. We will assume that it is an analytic function at $y \rightarrow 0$ with the characteristic scale $L$; non-analytic forms of $\kappa$ have been considered as well [1]. This model has attracted considerable attention since it is believed to be exactly solvable $1,2,43,4,5,6$, 7, 8, 9, 10, 11, 12, 13, 14.

Let us introduce the characteristic function of the $N$ point velocity distribution, $Z_{N}\left(\lambda_{1}, x_{1} ; \ldots ; \lambda_{N}, x_{N} ; t\right)=$ $\left\langle\exp \left(\lambda_{1} u\left(x_{1}, t\right)+\ldots+\lambda_{N} u\left(x_{N}, t\right)\right)\right\rangle$. As was shown in [2], it obeys the master equation that one derives by differentiating this function with respect to $t$, and by using Eq. (1),

$$
\frac{\partial Z_{2}}{\partial t}=-\lambda_{i} \frac{\partial}{\partial \lambda_{i}} \frac{1}{\lambda_{i}} \frac{\partial Z_{2}}{\partial x_{i}}+\frac{1}{2} \lambda_{i} \lambda_{j} \kappa\left(x_{i}-x_{j}\right) Z_{2}+D
$$

where the summation over repeated indices is assumed.

The last term in Eq. (3) denotes the contribution of the dissipative terms. Although the method of our paper is valid for the general case of $N$-point characteristic function, we will concentrate on the case $N=2$, where the solution is easy to find. In this case, $D=$ $\nu\left\langle\left(\lambda_{1} u_{x_{1} x_{1}}+\lambda_{2} u_{x_{2} x_{2}}\right) \exp \left(\lambda_{1} u\left(x_{1}, t\right)+\lambda_{2} u\left(x_{2}, t\right)\right)\right\rangle$. This term does not vanish in the limit of infinitely large Reynolds number, $\nu \rightarrow 0$; rather, it has a finite value, because the velocity field develops singularities, shocks, where large velocity gradients are balanced by the small viscosity. It has been proposed in 22] that the origin of this term is analogous to the origin of anomalies in quantum field theories, and it has been suggested that this term can be expressed linearly through the $Z$ function. Namely, the following ansatz should be true inside any
$N \geq 2$ point correlation function

$$
A \equiv \lim _{\nu \rightarrow 0} \nu \lambda u_{x x} e^{\lambda u(x)}=\left[\frac{a}{2}+\frac{b-1}{\lambda} \frac{\partial}{\partial x}\right] e^{\lambda u(x)} .
$$

The right hand side of (4) contains the simplest linear in $e^{\lambda u}$ terms that are consistent with translation, scale, and Galilean invariance 2, 3]. The higher-derivative terms are not allowed since they would change the structure of Eq. (3) and may lead to additional, non-physical, solutions. The parameters $a$ and $b$, which we will call 'anomalies,' should be found from the requirement that the stationary solution of Eq. (3) correspond to a positive, finite, and normalizable PDF, similar to the eigenvalue problem in quantum mechanics. The other condition is non-positivity of the dissipation, $D \leq 0$. These two conditions restrict the values of the anomalies considerably. It has been shown in 3 that the admissible solution exists for a one-parameter family $\{b, a(b)\}$, where $3 / 4 \leq b \leq 1$ and the corresponding interval for the $a$ anomaly is $0 \geq a \geq-0.45$.

The corresponding scale-invariant solution for the velocity difference PDF, $w(\Delta u / y)$, where $\Delta u=u\left(x_{1}\right)-$ $u\left(x_{2}\right) \ll u_{r m s}$ and $y=x_{1}-x_{2} \ll L$ can be found from (3). It has a peculiar structure that was first qualitatively established in numerical simulations [1] and was confirmed by a variety of analytical methods 2 , 3, 4, 5, 6, 7]. It decays hyper-exponentially fast for the large positive argument, $w(z) \propto \exp \left(-z^{3} / 3\right)$, and has an algebraic tail for the large negative argument, $w(z) \propto|z|^{-2 b-1}$ [see section 2]. This behavior is understood since due to the nonlinear term in Eq. (11), positive gradients decrease in the course of time, while negative gradients become stronger. Therefore, large negative velocity gradients are more probable than large positive ones.

According to the allowed values of the anomaly $b$ in model (4), the distribution density should decay slower than $|z|^{-3}$ for $z \rightarrow-\infty$. However, the geometric consideration of pre-shock events producing large negative velocity gradients, proposed in [7, 8], suggested the asymptotic $|z|^{-7 / 2}$. Lagrangian simulations of only the left tail of the PDF agreed with the latter result [12, 13, 14]. 
So far, no analytical derivation of the full velocitydifference PDF decaying as $|z|^{-7 / 2}$ was available. The direct numerical simulations of Eq. (1), carried out in [9, 10], have not convincingly reproduced such a PDF either. The form of the PDF has therefore remained a subject of controversy.

In this Letter we propose that the PDF is not unique. In an infinite system, where the Galilean invariance holds, the anomaly has the form (4). In the finite-size systems, investigated in [12, 13, 14], the global Galilean invariance is broken and the weak Galilean principle should be applied. In this case the form of the anomaly is different from (44), and the solution with the asymptotics $|z|^{-7 / 2}$ may be allowed. We derive the corresponding velocity and velocity-difference PDFs. Next, we conduct extensive numerical simulations of Eq. (10), using a highresolution shock capturing scheme. The results agree well with our analytical prediction. Section 2 presents the theory, section 3 - numerical simulations.

2. Velocity and velocity-difference PDF's. The resolution of the controversy mentioned above is based on the fact that the form (4) of the anomaly is dictated by the strong form of the Galilean principle (see below) while the arguments of [7, 8] as well as numerical results 12, 13, 14] are correct only when the weak G-principle applies. The strong Galilean principle is the requirement that in the infinite system, taking velocity to zero at infinity does not break the Galilean symmetry in the middle. This requirement is analogous to the absence of the spontaneous symmetry breaking in magnetic systems. For the systems considered in 7, 8, 12, 13, 14] this strong Gprinciple obviously does not hold. The reason is that the size of the systems is of the same order as the correlation length of the force. Therefore, the velocities in the middle of the system are correlated to those at the boundary. The same argument works for the periodic case, in which the Galilean symmetry is broken by the condition $u_{0}=\int u d x=0$.

So, the results in 2] and 7, 8, 12, 13, 14] refer to the different dynamical systems and that explains the discrepancy. The natural question is whether we can apply the methods of [2] for the case in which the strong G-symmetry is broken. This is important because the method of dissipative anomalies is the only known nonperturbative way to solve Burgers turbulence. In this case we still have the weak G-symmetry, which says that all correlation functions are G-symmetric, provided that we average them over $u_{0}$. This is of course always true, but how to impose this condition?

Let us notice that the correlation functions calculated at the fixed $u_{0}$ have the property

$$
\left\langle e^{\sum \lambda_{j} u\left(x_{j}, t\right)}\right\rangle_{u_{0}}=e^{u_{0} \sum \lambda_{j}}\left\langle e^{\sum \lambda_{j} u\left(x_{j}, t\right)}\right\rangle_{0} .
$$

Hence, if we integrate over $u_{0}$ the correlations must have the form:

$$
\left\langle e^{\sum \lambda_{j} u\left(x_{j}, t\right)}\right\rangle=2 \pi \delta\left(\sum \lambda_{j}\right)\left\langle e^{\sum \lambda_{j} u\left(x_{j}, t\right)}\right\rangle_{0} .
$$

The master equation (3) must allow this ansatz, namely the proportionality to the $\delta\left(\sum \lambda_{j}\right)$ must be consistent with the equation. This is obviously so with the anomaly (4), but this also allows a more general form of the anomaly. Let us look at the expression

$$
\tilde{A}=A+c \lambda \frac{\partial}{\partial \lambda} e^{\lambda u}
$$

it adds the term $\tilde{D}=c \sum \lambda_{j} \partial Z / \partial \lambda_{j}$ to the right hand side of Eq. (3). When $Z=\delta\left(\sum \lambda_{j}\right) Z_{0}$, we have $\tilde{D}=$ $c\left(\sum \lambda_{j} \partial Z_{0} / \partial \lambda_{j}-Z_{0}\right) \delta\left(\sum \lambda_{j}\right)$, where we used the identity $x \delta^{\prime}(x)=-\delta(x)$. It is important to notice that any other function of $\lambda$ or higher power of $\partial / \partial \lambda$ would break the ansatz. Anomalies of the form (7) have been considered in relation to incompressible turbulence by $\mathrm{V}$. Yakhot [private communication].

The restrictions on the coefficients $a, b$, and $c$ can be obtained from the equation for the velocity-difference PDF. To write this equation, substitute the dissipative term (77) into Eq. (31). In the Galilean-invariant limit, the statistics of velocity differences are separated from those of the mean velocity field. Let us change the variables, $\lambda_{1}=\Lambda+\mu, \lambda_{2}=\Lambda-\mu, x_{1}=X+y / 2, x_{2}=X-y / 2$, and assume that $\Lambda \ll \mu$, and $y \ll L$. The latter condition allows us to expand $\kappa(y) \simeq \kappa_{0}-\kappa_{2} y^{2} / 2$. In this limit, the master equation (33) becomes

$$
\begin{array}{r}
\frac{\partial^{2} Z_{2}}{\partial \mu \partial y}-\frac{2 b}{\mu} \frac{\partial Z_{2}}{\partial y}-2 \Lambda^{2} \kappa_{0} Z_{2}-\frac{\kappa_{2} \mu^{2} y^{2}}{2} Z_{2} \\
=c \mu \frac{\partial Z_{2}}{\partial \mu}+c \Lambda \frac{\partial Z_{2}}{\partial \Lambda}+a Z_{2}
\end{array}
$$

where we consider the stationary case, $\partial_{t} Z_{2}=0$. In the finite-size system with $u_{0}=0$, we assume the weak Gsymmetry and look for the solution in the form $Z_{2}=$ $Z_{+}(\Lambda) Z_{-}(\mu, y)$. [The strong G-symmetry would imply $Z_{+}(\Lambda) \sim \delta(\Lambda)$ and the solution might be different.] Substituting this into Eq. (8) we get the following set of equations for the characteristic functions $Z_{+}$and $Z_{-}$:

$$
\begin{array}{r}
-2 \Lambda^{2} \kappa_{0} Z_{+}=c \Lambda \frac{\partial Z_{+}}{\partial \Lambda}, \\
\frac{\partial^{2} Z_{-}}{\partial \mu \partial y}-\frac{2 b}{\mu} \frac{\partial Z_{-}}{\partial y}-\frac{\kappa_{2} \mu^{2} y^{2}}{2} Z_{-}=c \mu \frac{\partial Z_{-}}{\partial \mu}+a Z_{-} .
\end{array}
$$

The solution of Eq. (9) corresponds to a positive and normalizable PDF only when $c<0$, and the solution is a Gaussian. Its Laplace transform then gives the velocity probability density function

$$
P(U)=\sqrt{\frac{-c}{\pi \kappa_{0}}} \exp \left(\frac{c U^{2}}{\kappa_{0}}\right),
$$


where $U=\left[u\left(x_{1}\right)+u\left(x_{2}\right)\right] / 2$. Since the point separation is much smaller than the force correlation length, $P(U)$ becomes the one-point probability density function of the velocity field. Strictly speaking, expression (11) is phenomenological, it is expected to match the velocity PDF only in the region $U \ll U_{r m s}$; for larger $U$ the velocity PDF may be not universal. We compare this result with the simulations in section 3 .

Equation (10) is more complicated, but it can be simplified if we are looking for the solution in the scaleinvariant form, $Z_{-}(\mu, y)=\Phi(x)$, where $x=\mu y$,

$$
x \Phi^{\prime \prime}+(1-2 b) \Phi^{\prime}-\frac{\kappa_{2}}{2} x^{2} \Phi=a \Phi+c x \frac{\partial}{\partial x} \Phi .
$$

To rewrite this equation in the velocity space, let us Laplace transform the $\Phi$ function,

$$
\Phi(x)=\int_{-\infty}^{+\infty} w(\tilde{z}) \exp (x \tilde{z}) d \tilde{z},
$$

The function $w(\tilde{z})$ is then related to the velocitydifference probability density function, $W(\Delta u, y)$, as $W(\Delta u, y)=w(\Delta u / y) / y$. Equation for $w$ is readily obtained by substituting (13) into (12). Introducing the dimensionless variable $z=\tilde{z}\left(\kappa_{2} / 2\right)^{-1 / 3}$, we thus get

$$
w^{\prime \prime}+z^{2} w^{\prime}+(1+2 b) z w=-\tilde{a} w+\tilde{c}(z w)^{\prime},
$$

where the derivatives are with respect to $z$, and $\tilde{a}=$ $a\left(\kappa_{2} / 2\right)^{-1 / 3}$ and $\tilde{c}=c\left(\kappa_{2} / 2\right)^{-1 / 3}$. Asymptotics of the solution at $|z| \rightarrow \infty$ can be found from Eq. (14),

$$
\begin{aligned}
w & \sim z^{(2 b-1)} \exp \left(-z^{3} / 3+\tilde{c} z^{2} / 2\right), \quad z \rightarrow+\infty, \\
w & \sim|z|^{-(2 b+1)}, \quad z \rightarrow-\infty
\end{aligned}
$$

they have the form that we already discussed in the introduction.

Ideally, we should be able to derive all the anomalies $a, b$, and $c$ from our theory. At present we are not able to do this, so in the next section we turn to numerical simulations.

In general, the anomalies $a, b$ and $c$ should satisfy the requirement that Eq. (14) have positive and normalizable solution, and that the dissipation is non-positive, $\tilde{a} w+$ $2(b-1) z w-\tilde{c}(z w)^{\prime} \leq 0[3]$. This eigenvalue problem thus has two-parameter family of solutions.

Note that the geometric approach of 7, , $8,11,12,13$, 14], proposed the asymptotic for negative velocity differences, $w \sim|z|^{-7 / 2}$. This condition is satisfied by $b=5 / 4$. This value was not allowed by the condition of nonpositivity of the dissipation in the theory based on (4), but with the new expression for the anomaly (7) this value is admissible. For $b=5 / 4, \mathrm{Eq}$, (14) can be solved numerically, leading to the approximate window of acceptable values, $-0.37 \leq \tilde{c} \leq-0.025$, which turns out to be consistent with (11). We now find the values of the anomalies from numerical simulations of Eq. (11).

3. Numerical results. We perform numerical simulations of Eq. (1) in a periodic interval of length $L=1$ containing $10^{6}$ grid points. The used numerical scheme is the standard shock capturing, total variation diminishing (TVD) scheme with non-linear, limited solution reconstruction 15]. For non-linear, scalar equations the scheme is provably TV-stable, monotonicity preserving and second-order accurate in the $L_{p}, p<\infty$, norm. To resolve discontinuous solutions the scheme uses nonlinear numerical viscosity that appears only inside the shocks. This dissipation mechanism ensures that the shocks are restricted to 2-3 grid points.

The external force is generated as

$$
f(x)=\sum_{k=1}^{m} A(k)\left[\xi_{k} \sin (2 \pi k x)+\eta_{k} \cos (2 \pi k x)\right],
$$

where $\xi_{k}$ and $\eta_{k}$ are independent Gaussian random variables with mean 0 and covariance 1 . The amplitude of the force is $A(k)=k\left[\exp \left(-k^{2} / n^{2}\right) / \tau\right]^{1 / 2}$, where $\tau$ is the force renewal time; in our simulations we had $\tau=10^{-5}$, while the integration time step was $t_{0}=10^{-7}$. We used $m=10$, and performed a series of runs for $n=5,10$. In this paper we mainly present the results averaged over 5 independent runs with $n=5$, and with the integration time $t=2$ each.

We found that the one-point PDF can be best matched by the solution of Eq. (11), with the choice $\tilde{c}=-0.3$. Formula (11) gives

$$
P(U)=\sqrt{\frac{0.3 \kappa_{2}^{1 / 3}}{2^{1 / 3} \pi \kappa_{0}}} \exp \left(-\frac{0.3 \kappa_{2}^{1 / 3}}{2^{1 / 3} \kappa_{0}} U^{2}\right),
$$

and Fig. (11) shows its good agreement with the simulations for both $n=5$ and $n=10$. As expected, the best agreement is seen in the top parts of the curves; the tails of the PDF seem to be non-universal.

Once the $c$ anomaly is given, we find the anomaly $b$ by matching the numerically obtained velocity-difference PDF with the solution of Eq. (14), see Fig. (2) 3). The best match to the whole PDF is given by $b \approx 1.19$ which is slightly less than the value $b=5 / 4$, predicted in 7, 8, 11, 12, 13, 14], and leads to the left-tail exponent -3.38 . The solution of Eq. (14) with the value $b=5 / 4$ is less consistent with our simulations. Although the reason for this discrepancy is quite intriguing and is not clear to us, our numerical resolution is not high enough to distinguish between the two asymptotics, -3.5 and -3.38 . Therefore, more extensive numerical work is required to understand this difference.

Once the anomalies $c$ and $b$ are found, the $a$ anomaly is obtained from the eigenvalue problem (14), which gives $\tilde{a} \approx-0.65$. 


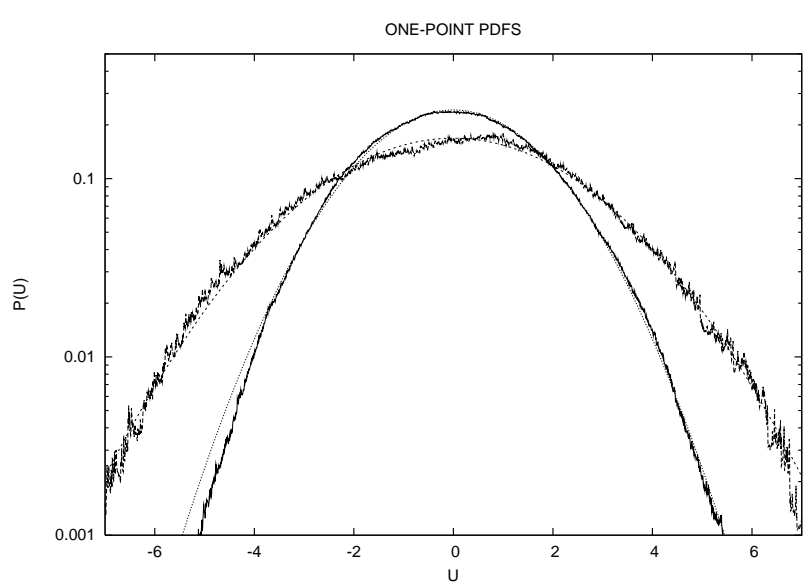

FIG. 1: Numerical and analytical one-point PDFs for two runs, $n=5$ and $n=10$. The broader curves correspond to the run $n=10$. The plot is in log-lin scale.

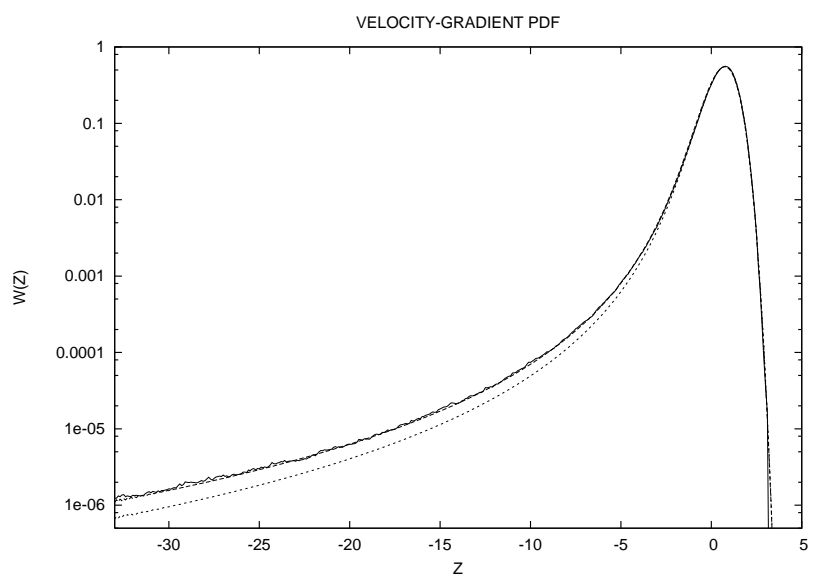

FIG. 2: Velocity-gradient PDF for the run $n=5$. The analytical curves correspond to the solution of Eq. (14) with $b=1.19$ (dashed line) and $b=5 / 4$ (dotted line). In both cases, $\tilde{c}=-0.3$. The plot is in log-lin scale.

4 Conclusions. We have found that our analytical model (14) with parameters $\tilde{a} \approx-0.65, b \approx 1.19$, and $\tilde{c} \approx-0.3$ provides an excellent fit to the numerically obtained velocity and velocity-difference PDF's of Burgers turbulence. As the important numerical check, one can directly find the form of the anomalies by numerically constructing conditional probabilities of shock amplitudes for given velocity gradients on the left and on the right of the shock [8]. We plan to address this question in the future with more extensive simulations.

We also believe that the method of dissipative anomalies can be generalized for turbulence with pressure and can be applicable in more than 1 dimension. We currently conduct the corresponding simulations, the results will be reported elswere. Practically, the Burgers model is expected to work for strongly compressible flows, i.e., in astrophysical application, such as supersonic turbu-

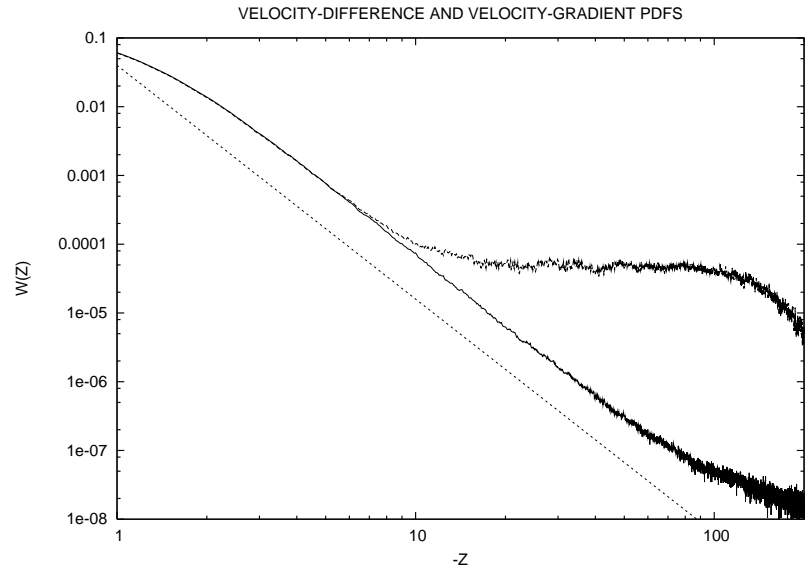

FIG. 3: The left tails of the velocity-gradient PDF (lower curve) and of the velocity-difference PDF (upper curve, point separation $\Delta x=10^{-3}$ ). For reader's orientation, the straight line has the slope -3.4 .

lence in cold molecular clouds [16, 17].

We thank Victor Yakhot for important discussions. The work of SB and TL was supported by the NSF Center for magnetic self-organization in laboratory and astrophysical plasmas at the University of Chicago. The research of AP was supported in part by the NSF grants PHY-9802484 and PHY-0243680.

[1] A. Chekhlov \& V. Yakhot, Phys. Rev. E 52 (1995) 5681; V. Yakhot \& A. Chekhlov, Phys. Rev. Lett. 77 (1996) 3118.

[2] A. Polyakov, Phys. Rev. E 52 (1995) 6183.

[3] S. Boldyrev, Phys. Rev. E 55 (1997) 6907; S. Boldyrev, Phys. of Plasmas, 5 (1998) 1681.

[4] V. Gurarie \& A. Migdal, Phys. Rev. E 54 (1996) 4908; V. Gurarie, hep-th/9606089

[5] J.-P. Bouchaud \& M. Mézard, Phys. Rev. E 54 (1996) 5116.

[6] E. Balkovsky, G. Falkovich, I. Kolokolov, \& V. Lebedev, Phys. Rev. Lett. 78 (1997) 1452.

[7] W. E, K. Khanin, A. Mazel, \& Ya. Sinai, Phys. Rev. Lett. 78 (1997) 1904.

[8] W. E \& E. Vanden Eijnden, Phys. Rev. Lett. 83 (1999) 2572; Comm. Pure Appl. Math. 53 (2000) 852.

[9] T. Gotoh \& R. H. Kraichnan, Phys. Fluids 10 (1998) 2859.

[10] T. Gotoh, Phys. Fluids 11 (1999) 2143.

[11] R. H. Kraichnan, chao-dyn/9901023

[12] J. Bec, Phys. Rev. Lett. 87 (2001) 104501.

[13] U. Frisch, \& J. Bec, in Les Houches 2000: New Trends in Turbulence; M. Lesieur, A. Yaglom and F. David, eds. (Springer EDP-Sciences, 2001), pp. 341-383.

[14] V. Gurarie, nlin.CD/0307033

[15] R. J. LeVeque, Numerical Methods for Conservation Laws, (Birkhäuser Verlag, Basel, 1992).

[16] C. F. von Weizsäcker, Astrophys. J. 114 (1951) 165. 
[17] C. M. Brunt, M. H. Heyer, E. Vázquez-Semadeni, \& B. Pichardo, ApJ 595 (2003) 824. 